\title{
Biocatalytic Redox Reactions with Allylic Alcohols
}

\author{
Luiz H. F. Araujo*, Fábio D. Nasário, Paulo J. S. Moran, J. Augusto R. Rodrigues.
}

\begin{abstract}
This work focused on the biocatalitic multi-enzymatic cascade reactions of allylic alcohols mediated by Candida albicans (CCT 0776), as well as the relation between the degree of substitution at the olefin and the enantioselectivity presented by the microorganism.
\end{abstract}

\section{Key words: \\ Biocatalysis, bioreduction, biotransformation.}

\section{Introduction}

The asymmetric hydrogenation reactions have critical roles in organic synthesis and in overall synthesis. Taking it into account, novel approaches have been in development, which consists in using enzymes to perform an enantioselective reduction of substituted double bonds. Such method is an alternative hydrogenation without employing molecular hydrogen and a metal catalyst, producing compounds containing a stereogenic center at $\alpha$ or $\beta$-carbon ${ }^{1}$. The present work focuses on observing both chemo and stereoselectivity of the microorganism Candida albicans in biocatalysis with allylic alcohols analogous to cinnamyl alcohol.

\section{Results and Discussion}

The study intended to be initiated with the biotransformation of $(E)-3$ and (Z)-3 alcohols. Their chemical synthesis was performed by a HWE reaction with acetophenone as a substrate, followed by a reduction with DIBAL-H ${ }^{2}$. The mixture $(E, Z)-3$ was purified in flash chromatography system, Isolera One - Biotage, obtaining a pure fraction only of $(E)-3$. It was not possible with this method to isolate $(Z)-3$.

The biocatalysis studies were carried out by using C. albicans cells as a biocatalyst and the substrate $(E)-3$ was used on the reaction in three conditions: free, supported on hydrophobic resin (XAD7HP) and supported on cellulose (small pieces of filter paper $-0,5 \mathrm{~cm}^{2}$ ). The substrate supporting is important because it slows the substrate release rate on the reaction media, avoiding any toxic effect on the microorganism caused by its high concentration. The $(E)-3$ biocatalysis was accompanied by fourteen days and the conversion data were obtained by GC-MS.

At the first $4 \mathrm{~h}$ it was observed that the $(E)-3$ conversion was higher when free or supported on paper compared to supported on resin, but it was not possible to determinate the selectivity towards any product in all conditions yet. After one day, the substrate supported on resin was completely converted into products, obtaining the compound $(S)-6(79 \%, 99 \%$ ee). In $2-5$ days, there were not changes in the produced compounds in all three conditions evaluated. However, in 14 days, it was noted a high conversion for the compound $\mathbf{8}, 88 \%$, with the resin support. Based on that, it was possible to point out that the substrate adsorption resin made the process more selective, giving mainly compound $(S)-6$ after one day and 8 after 14 days.

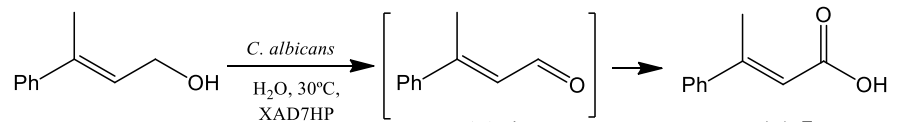

$(E)-3$ XAD7HP (E)-7<smiles>C[C@H](CCO)c1ccccc1</smiles>

(S)-6
$(E)-4$

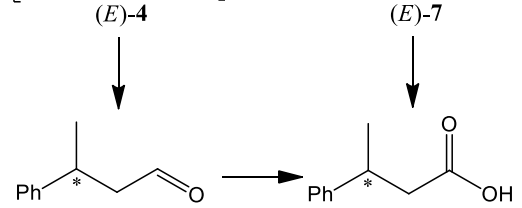

8
Image 1. Reaction sequence proposed to the biocatalysis with $C$. albicans of the $(E)-3$ supported on resin.

Although it was not possible to obtain a pure fraction of (Z)-3 with the procedure described previously. A new method was found to synthesize after three steps with $70 \%$ yield $^{3}$. The next step is performing the biocatalysis of $(Z)-3$, with $C$. albicans and evaluate the difference on the products stereochemistry compared to $(E)-3$.

\section{Conclusions}

The biocatalytic study with $(E)-3$ presented interesting selectivity results when it was adsorbed on resin. After one day, it was observed $79 \%$ of $(S)-6$ with $99 \%$ ee. After 14 days, it was pointed out the oxidative capacity of $C$. albicans enzymes, since most of the saturated alcohol (S)-6 was converted to the saturated acid $\mathbf{8}$. Therefore, it is a green methodology, intrinsically safe to promote hydrogenation and oxidation in cascade to produce enantiomerically enriched alcohol and acid from the allylic alcohol, without metal catalyst like $\mathrm{Ru}, \mathrm{Rh}$, and high pressure of $\mathrm{H}_{2}{ }^{4}$. Besides that, the formation of acid from the allylic alcohol was unexpected once this kind of transformation is scarce in literature.

\section{Acknowledgement}

To FAPESP (Grant 2016/12074-7), PIBIC/CNPq and Institute of Chemistry UNICAMP.

\footnotetext{
${ }^{1}$ Nestl, B. M.; Hammer, S. C.; Nebel, B. A.; Hauer, B. Angew. Chem., Int. Ed. 2014, 53, 3070.

${ }^{2}$ Li, J.; Peters, B.; Andersson, P. Chem. Eur. J., 2011, 17, 11143

3 Fananas-Mastral, M.; Vitale, R.; Perez, M.; Feringa, B. L., Chemistry 2015, 21 (11), 4209-12.

${ }^{4}$ Wu, R.; Beauchamps, M. G.; Laquidara, J. M.; Sowa, J. R. Angew. Chem., Int. Ed. 2012, 51, 2106.
} 